Demokratie-Lernen

als politische

und pädagogische

Aufgabe 


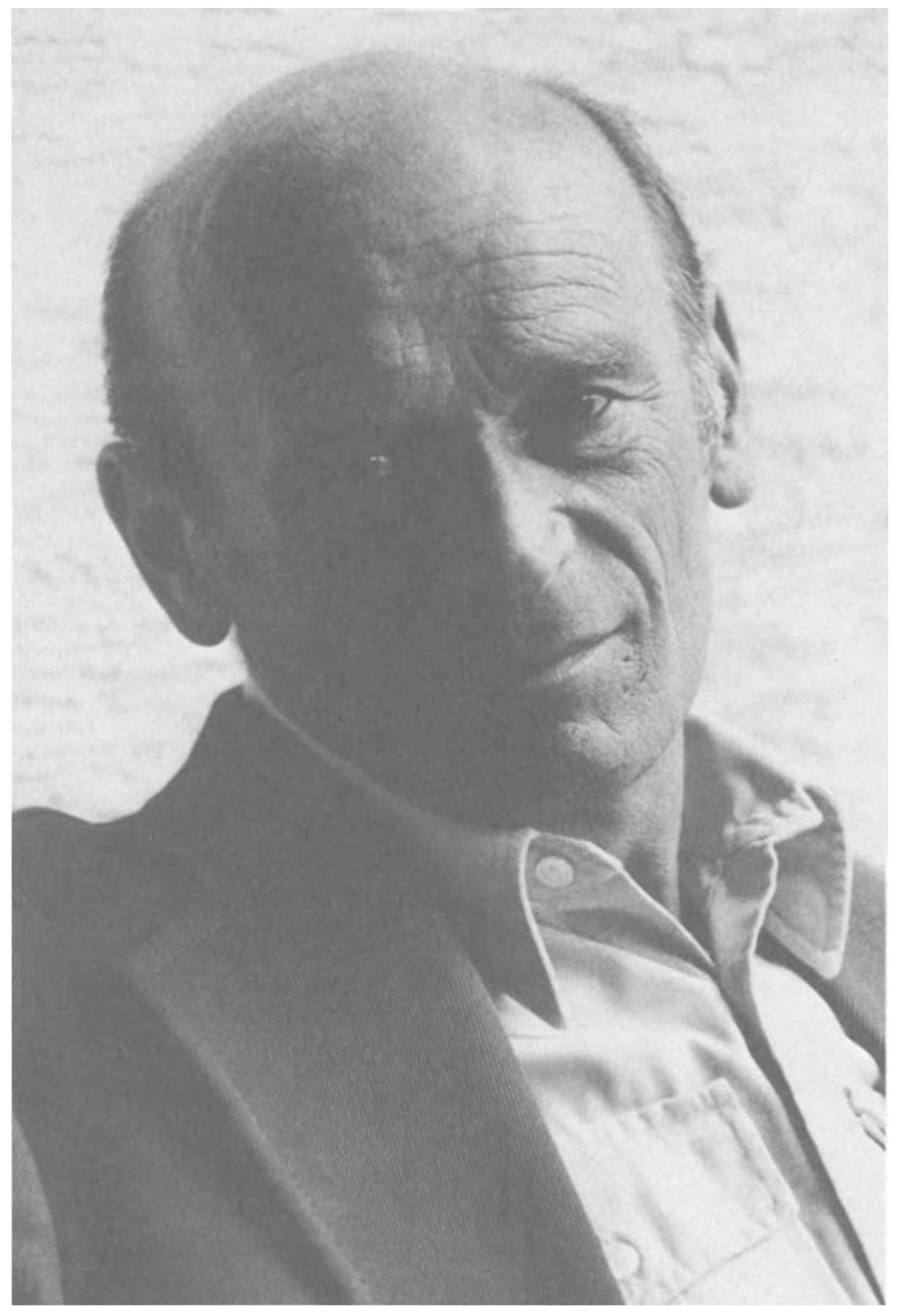

KURT GERHARD FISCHER 


\title{
Demokratie-Lernen als politische und pädagogische Aufgabe
}

Für Kurt Gerhard Fischer zum 60. Geburtstag
herausgegeben
von Siegfried George
und Wolfgang Sander

\author{
J.B. Metzlersche \\ Verlagsbuchhandlung \\ Stuttgart
}


CIP-Titelaufnahme der Deutschen Bibliothek

Demokratie-Lernen als politische und pädagogische Aufgabe : für Kurt Gerhard Fischer zum 60. Geburtstag / hrsg. von Siegfried George u. Wolfgang Sander. - Stuttgart : Metzler, 1988

ISBN 978-3-476-30297-7

NE: George, Siegfried [Hrsg.]; Fischer, Kurt Gerhard: Festschrift

ISBN 978-3-476-30297-7

ISBN 978-3-476-99050-1 (eBook)

DOI 10.1007/978-3-476-99050-1

Dieses Werk einschließlich aller seiner Teile ist urheberrechtlich geschützt. Jede Verwertung außerhalb der engen Grenzen des Urheberrechtsgesetzes ist ohne Zustimmung des Verlages unzulässig und strafbar.

Das gilt insbesondere für Vervielfältigungen, Übersetzungen, Mikroverfilmungen und die Einspeicherung und Verarbeitung in elektronischen Systemen.

(C) 1988 Springer-Verlag GmbH Deutschland Ursprünglich erschienen bei J.B. Metzlersehe Verlagsbuchhandlung und Carl Ernst Poeschel Verlag GmbH in Stuttgart 1988

Verlagsredaktion: Rosemarie Flory 


\section{Inhalt}

\section{Vorwort}

RUDOLF ENGELHARDT

Ein Besuch bei Kurt Gerhard Fischer 1

WOLFGANG KLAFKI

Theodor Litt zwischen Weimar und Bonn 13

CLEMENS LESSING

Politisches Handeln für die

Politische Bildung - Zur Geschichte der

Deutschen Vereinigung für Politische Bildung 41

BJÖRN ENGHOLM

Der Politiker als Anwalt der Politischen Bildung 55

WILL CREMER

Politikwissenschaft und Politische Bildung

Zur Intention der didaktischen Fachtagungen

der Bundeszentrale für Politische Bildung 65

WOLFGANG HILLIGEN

Das Schulbuch als Pädagogicum und Politicum 85

KURT F. K. FRANKE

Völkerverständigung durch

historisch-politische Bildung -

Deutsche Nachkriegsgeschichte

in ausgewählten italienischen Schulgeschichtsbüchern 113 
RAINER KRIEGER

Schüler urteilen über Jugendarbeitslosigkeit oder: Vom Ankreuzen zum Artefakt 137

ULRICH HAIN

Kunde und Wissenschaft - Politische Bildung von außen 153

VOLKER NITZSCHKE

Methodisches Handeln im politischen Unterricht.

Versuch einer begrifflichen Klärung und Begründung 165

DIETRICH ZITZLAFF

Die Kinder- und Jugendliteratur der DDR -

Voller Anregungen für die Politische Bildung 183

MICHELE BORRELLI

Interkulturelle Pädagogik als Wissenschaft der Erziehung 205

WOLFGANG SANDER

Die Einheit der Politischen Bildung.

Zusammenhang und Unterscheidung von Politischer Bildung in Schule und Erwachsenenbildung 233

SIEGFRIED GEORGE

Politisch-pädagogische Reflexionen über Neue Medien 247

Verzeichnis der Schriften von Kurt Gerhard Fischer 267

Autorenverzeichnis 283 


\section{Vorwort}

Kurt Gerhard Fischer mag keine Festschriften, will keine Belobigungen. Ein themenorientiertes Buch ist ihm lieber. Deshalb haben wir uns entschlossen, ein Sachbuch zur Politischen Bildung zu schaffen, das zwar auf das Werk von Fischer Bezug nimmt, aber eine allgemeine Thematik behandelt.

Dokument für das umfangreiche Schaffen von Fischer ist die Liste seiner Veröffentlichungen am Ende dieses Bandes. Wie dort leicht ersichtlich, waren und sind seine Interessen nicht eng fachbezogen. Seine Arbeiten als Schriftsteller und Editor sind ebenso ein Ausdruck seines Lebens wie die Veröffentlichungen zur Politischen Bildung. Geprägt von der Erfahrung des Nationalsozialismus, ist es immer eine Maxime von Fischers pädagogischen Arbeiten - seiner Theoriebeiträge ebenso wie seiner Schulbücher - gewesen, daß Schule dazu beitragen möge, einen erneuten "Rückfall in die Barbarei« zu verhindern. Er selbst hat einmal "Demokratie-Lernen" als die "Generalaufgabe der Schule" bezeichnet.

Es ist unmöglich, auch nur annähernd die Wirkung von Kurt Gerhard Fischer auf die Entwicklung der Politischen Bildung in Deutschland und anderen Ländern zu skizzieren. Viele Autoren haben sich mit seinen Schriften auseinandergesetzt, teils zustimmend, teils ablehnend gelegentlich jedoch ohne die erforderliche intellektuelle Redlichkeit.

Eine Festgabe ist ein Ausdruck von Respekt, Dank und Freundschaft. Sie gilt dem Autor der Schriften, mehr noch dem Menschen in seinen vielfältigen Lebensbezügen. Fischer ist ein Mensch mit Ecken und Kanten. Seine ausgeprägte Individualität wird es ihm auch in Zukunft ermöglichen, ein erfülltes Leben zu haben.

Wir werden auch von seinem neuen Wohnsitz in der Toscana aus weiter von ihm hören. 
Unser besonderer Dank gilt den Autoren, die durch ihre spontane Bereitschaft, den Jubilaren auf diese Weise zu ehren, uns bestärkt haben, das Projekt zu realisieren. Dank gilt auch der J.B. Metzlerschen Verlagsbuchhandlung, die diese Festschrift einer breiten Öffentlichkeit zugänglich machen will.

SIEGFRIED GEORGE

WOLFGANG SANDER 